\title{
Mechanisms of human susceptibility to Burkholderiapseudomallei infection
}

\section{Introduction}

G Lertmemongkolchai ${ }^{1,2}$

Burkholderiapseudomallei infection (melioidosis) is an increasing public health burden in NE Thailand and severely under-reported in other countries worldwide. This organism is a significant bio-threat agent and a facultative intracellular pathogen.Type 2 diabetes mellitus is the most common risk factor for melioidosis.

\section{Objectives}

To characterise human immune responses to $B$. pseudomallei in healthy persons versus patients with diabetes mellitus living in an endemic area of melioidosis.

\section{Methods}

Human neutrophil functions were studied by means of phagocytosis, killing by oxidative burst, migration, apoptosis and cytokine productions.

\section{Results\& Discussion}

Human neutrophil responses were impaired in individuals with diabetes mellitus. More than half of the diabetic patients were prescribed glibenclamide (also known as glyburide) to control blood glucose levels. Recent evidence demonstrated that glibenclamide reduced pro-inflammatory cytokine production and migration capacity by neutrophils of diabetic individuals in response to this bacterial infection. Furthermore we found that glibenclamide decreased free glutathione levels and glutathione peroxidase of neutrophils after exposure to live B. pseudomallei. Interestingly exogenous glutathione could restore these functions.

\section{Conclusion}

Taken together, our data show a link between the effect of glibenclamide on glutathione and neutrophil functions in response to $B$. pseudomallei that may contribute to the susceptibility of diabetic individuals to $B$. pseudomallei infection.

${ }^{l}$ Department of Microbiology, Mekong Health Science Research Institute, Thailand ${ }^{2}$ Faculty of Associated Medical Sciences, Khon Kaen University, Khon Kaen, Thailand.

Address for correspondence: Dr Gan Lertmemongkolchai, Department of Microbiology, Mekong Health Science Research Institute, Thailand. +66897127604Email: g.lert@yahoo.co.uk

(D) https://orcid.org/0000-0001-6972-585X 